\title{
Modeling Methodology for Elastomer Dynamics *
}

\author{
H.T. Banks \\ Nancy Lybeck \\ Center for Research in Scientific Computation \\ North Carolina State University \\ Raleigh, NC 27695-8205
}

September 30, 1996

\begin{abstract}
As engineering applications of elastomers increase in complexity, knowledge of the behavior of these materials, and the ability to predict these behaviors, becomes increasingly valuable. Elastomers exhibit a complex variety of mechanical properties, including nonlinear constitutive laws, strong damping and hysteresis (loss of kinetic and potential energy, respectively), and the dependence of strain on its history. Most current models for rubber-like materials assume a form of the strain energy function (SEF), such as a cubic Mooney-Rivlin form or an Ogden form. While these methods can produce good results, they are only applicable to static behavior, and they ignore hysteresis and damping. We discuss a dynamic partial differential equation (PDE) formulation based on large deformation theory elasticity as an alternative approach to the SEF formulation. Models using the PDE formulation are presented for both simple extension and generalized simple shear.
\end{abstract}

\section{Introduction}

In recent years the use of rubber in engineering applications has expanded well beyond traditional products such as tires and belts. Today rubber (and, more generally, elastomers) is employed in a diverse set of applications including sealing, vibration damping, and load bearing (see [5, 9]). The applications of rubber are becoming increasingly sophisticated, as exemplified by the use of rubber and rubber-like polymers in building supports which protect the structure during an earthquake (see [7]).

*Invited lecture, 1996 Conference on Math Theory of Network and Systems (MTNS), June 24-28, St. Louis, Missouri 
Traditionally, elastomers are filled with carbon black or silica particles, inactive substances chosen to change the physical properties of the material to match the needs of the given application. A controllable elastomer, resulting from the addition of active fillers such as conductive, magnetic, or piezoelectric particles, could be used in products such as active vibration suppression devices. As these new composite materials are developed, the applications of rubber will become more complex, and design will play an even more prominent role in the development of components. As a consequence, the capability to predict the dynamic mechanical response of the components undergoing a variety of deformations will become most important.

Many complications arise in the process of formulating models for elastomers, some of which can be attributed to the desirable characteristics of rubber as a design component, including the ability to undergo large elastic deformations, good damping properties, and near incompressibility. For example, rubber components often undergo large deformations, hence infinitesimal based strain theory is not appropriate. Damping is highly significant, and the nonlinear constitutive laws cannot be modeled by Hooke's law. The mechanical response is affected in a nontrivial manner by environmental temperature, amount and type of filler, rate of loading, and strain history. Moreover, many elastomers, especially those with a synthetic rubber base, exhibit strong hysteresis characteristics similar to those found in shape memory alloys and piezoceramic materials.

Researchers have made substantial progress in developing elastomer models (see $[6,13,15]$ for basic texts), the majority of which are phenomenological, based on finite strain (FS) and strain energy function (SEF) theories. SEF theories are typically used for static finite element analysis (see [4]), as the strain energy functions contain information about the elastic properties of elastomers, but do not describe either damping or hysteresis. The SEF material models are based on the principal extension ratios $\lambda_{i}$ (a misnomer widely found in the literature - "principal stretches" is a more appropriate terminology) which represent the deformed length of unit vectors parallel to the principal axes (the axes of zero shear stress). Rivlin proposed ([12]) that the SEF should depend only on the strain invariants $I_{1}=\lambda_{1}^{2}+\lambda_{2}^{2}+\lambda_{3}^{2}, I_{2}=\lambda_{2}^{2} \lambda_{3}^{2}+\lambda_{1}^{2} \lambda_{3}^{2}+\lambda_{1}^{2} \lambda_{2}^{2}$ and $I_{3}=\lambda_{1}^{2} \lambda_{2}^{2} \lambda_{3}^{2}$. Many SEF models are founded on this assumption, including the Mooney SEF $U=C_{1}\left(I_{1}-3\right)+C_{2}\left(I_{2}-3\right)$, or more generally, the modified expression $U=C_{1}\left(I_{1}-3\right)+f\left(I_{2}-3\right)$, where $f$ has certain qualitative properties. This class of models is most appropriate for components where the rubber is not tightly confined and where the assumption of absolute incompressibility (implying $\lambda_{1} \lambda_{2} \lambda_{3}=1$ or $I_{3}=1$ ) is a reasonable approximation. The more general Rivlin SEF $U=\sum_{i+j \geq 1}^{N} C_{i j}\left(I_{1}-3\right)^{i}\left(I_{2}-3\right)^{j}$ and its generalization for near incompressibility (see [4]) permit higher order dependence of the 
SEF on the invariants. An important departure form Rivlin's proposal is found in the work of Ogden, as well as that of Valanis and Landel, where the models use strain energy functions that depend only on the extension ratios $[10,14]$.

The finite strain elastic theory of Rivlin $[12,15]$ is developed with a generalized Hooke's law in an analogy to infinitesimal strain elasticity but makes no "small deformation" assumption and includes higher order exact terms in its formulation. In addition, finite stresses are defined relative to the deformed body and hence are the "true stresses" as contrasted to the "nominal" or "engineering" stresses (relative to the undeformed body) one encounters in the usual infinitesimal linear elasticity employed with metals. This Eulerian measure of strain (relative to a coordinate system convected with the deformations) - as opposed to the usual Lagrangian measure (relative to a fixed coordinate system for the undeformed body) - is an important feature of any development of models for use in analytical/computation/experimental investigations of rubber-like material bodies. The finite strain elasticity of Rivlin can be directly related to the strain energy function formulations through equations relating the finite strains $\tilde{e}_{x_{1} x_{1}}, \tilde{e}_{x_{2} x_{2}}, \tilde{e}_{x_{3} x_{3}}$ to the extension ratios $\lambda_{1}, \lambda_{2}, \lambda_{3}$ used in the SEF. However, the finite strain approach can be formulated in a somewhat more general framework in the context of classical modeling of elastic solids and fluids. Unfortunately, elastomers and filled rubbers are not exactly in either category. In the next section, we present a general procedure for developing models using large deformation elasticity theory. This will be followed by examples on simple extension and generalized simple shear illustrating the use of this methodology in developing models.

\section{Modeling in large deformation elasticity}

In this section we outline approaches to modeling the dynamics of elastic bodies where large deformations are of primary interest. Rather detailed discussions can be found in the texts by Ogden [11] and MarsdenHughes [8]. For our discussions we consider the body, depicted in Figure 1, with reference configuration $\Omega_{0}$ in the fixed principal $\vec{X}$ coordinate system. Translations, rotations, and deformations are all possible results of applied body forces. Our primary interest is in deformations of this body. Let $\Omega$ be the current configuration of the (deformed) body in the principal $\vec{x}$ coordinate system, as depicted in Figure 2.

The Lagrangian or fixed coordinate system, $\vec{X}$, is appropriate for small displacements from which the body fully recovers. The Eulerian or moving coordinate system, $\vec{x}$, is appropriate for large deformations from which the body does not fully recover. Elastomers typically undergo large deformations from which they fully (or almost fully) recover. Since it is not always 
clear which coordinate system is best to use in elastomers, the ability to translate quantities between the Lagrangian coordinate system and those quantities in the Eulerian coordinate system is highly desirable, indeed essential.

Using a summation convention, one can write $\vec{x}=x_{i} \vec{e}_{i}$ and $\vec{X}=X_{i} \vec{E}_{i}$, where $\vec{e}_{i}$ and $\vec{E}_{i}$ are unit vectors parallel to the $x_{i}$ and $X_{i}$ axes, respectively. The $X_{i}$ are called "referential," or Lagrangian coordinates while the $x_{i}$ are called "current" or Eulerian coordinates of a point. We may define a "configuration," or "position," map $\vec{x}=\phi(\vec{X})$. A "motion" or "trajectory" for a particle initially at $\vec{X}$ is given by $\vec{x}(t)=\phi(t, \vec{X})$. The configuration map is sometimes also called the "deformation map" but this is a misnomer, as the map does not give the deformation

$$
\begin{aligned}
u(t, \vec{X}) & =\phi(t, \vec{X})-\phi(0, \vec{X}) \\
& =\phi(t, \vec{X})-\vec{X},
\end{aligned}
$$

as usually defined in elasticity (where the deformation is zero for an undeformed body). The Lagrangian description of a physical phenomenon associated with a deformation of a body involves vector and scalar fields defined over the reference configuration $\Omega_{0}$ while the Eulerian description involves quantities defined over $\Omega$.

In studying deformations, it is desirable to transform physical quantities defined over a region in the Lagrangian coordinate system to quantities over a region in the Eulerian coordinate system (and conversely). To this end we consider the "configuration gradient"

$$
A=\left(\frac{\partial x_{i}}{\partial X_{j}}\right)=\frac{\partial \vec{x}}{\partial \vec{X}}=\frac{\partial \phi(\vec{X})}{\partial \vec{X}}
$$

which is the nonsingular, nonsymmetric gradient of the "configuration" map. Here $d x_{i}=\frac{\partial x_{i}}{\partial X_{j}} d X_{j}$, so $d \vec{x}=A d \vec{X}$ and $A$ is literally the "change

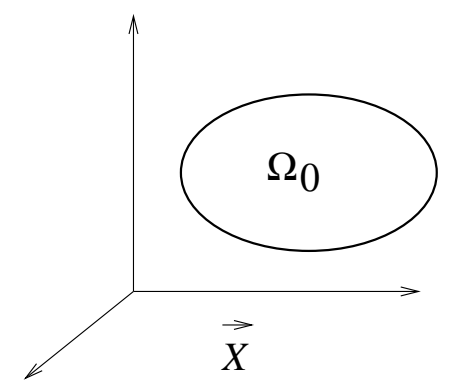

Figure 1: Reference configuration 


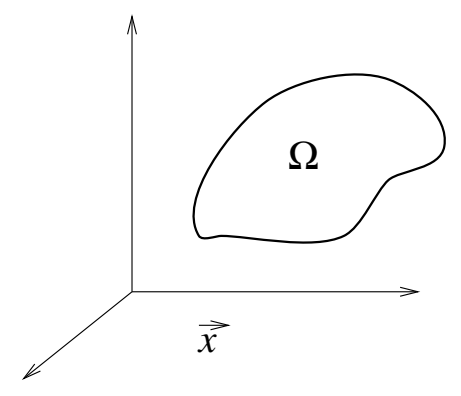

Figure 2: Deformed or current configuration

of configuration" gradient. Let $J=\operatorname{det} A, B=\left(A^{-1}\right)^{T}$. Then a standard elementary calculus result yields

$$
\int_{\Omega} d \omega=\int_{\Omega_{0}} J d \omega_{0}
$$

since volume elements in the Eulerian system are related to volume elements in the Lagrangian system via $d \omega=J d \omega_{0}$. For a volume-preserving (isochoric) material (a common approximation used in rubber based elastomers), $J=1$.

If the usual deformation $u(\vec{X})=\phi(\vec{X})-\vec{X}$ is considered, then $\frac{\partial u}{\partial \vec{X}}=A-$ $I$ is the actual deformation gradient. The matrix $A$ is sometimes termed the "deformation gradient," but this is misleading since our undeformed body thus has the identity as its "deformation gradient" if this terminology is adopted. The right Cauchy-Green tensor, defined by $A^{T} A=\left(\frac{\partial \vec{x}}{\partial \vec{X}}\right)^{T} \frac{\partial \vec{x}}{\partial \vec{X}}=$ $\left(\sum_{k} \frac{\partial x_{k}}{\partial X_{i}} \frac{\partial x_{k}}{\partial X_{j}}\right)$, and the left Cauchy-Green tensor, $A A^{T}=\left(\sum_{k} \frac{\partial x_{i}}{\partial X_{k}} \frac{\partial x_{j}}{\partial X_{k}}\right)$, are both symmetric, and play a fundamental role in strain analysis.

Given the above formulations there are several distinct ways to define a strain tensor. To begin with, we consider the change in squared lengths from the reference configuration to the current configuration

$$
|d \vec{x}|^{2}-|d \vec{X}|^{2}=d \vec{X} \cdot\left(A^{T} A-I\right) d \vec{X},
$$

and $A^{T} A-I$ is a measure of the strain (sometimes called the Lagrangian or Green strain). Thus, the body is unstrained if and only if $A^{T} A=I$.

One could then define the strain tensor

$$
E \equiv \frac{1}{2}\left(A^{T} A-I\right)
$$

so that (using a summation convention)

$$
E_{\alpha \beta}=\frac{1}{2}\left(A_{i \alpha} A_{i \beta}-\delta_{\alpha \beta}\right)
$$




$$
=\frac{1}{2}\left(\frac{\partial x_{i}}{\partial X_{\alpha}} \frac{\partial x_{i}}{\partial X_{\beta}}-\delta_{\alpha \beta}\right) .
$$

In elasticity one usually finds more terms in the strain tensor since it most often is defined in terms of the deformations instead of configurations. If one considers $u(\vec{X})=\phi(\vec{X})-\vec{X}$, then the true "deformation gradient" is given by

$$
D \equiv \frac{\partial u}{\partial \vec{X}}=A-I
$$

and hence

$$
A^{T} A-I=D^{T} D+D+D^{T} .
$$

Substituting into the definition of strain, we find that

$$
E_{\alpha \beta}=\frac{1}{2}\left(\frac{\partial u_{i}}{\partial X_{\alpha}} \frac{\partial u_{i}}{\partial X_{\beta}}+\frac{\partial u_{\alpha}}{\partial X_{\beta}}+\frac{\partial u_{\beta}}{\partial X_{\alpha}}\right) .
$$

This is the usual Green strain found in most elasticity books.

The above definitions and formulations involve static concepts. We are interested in dynamics of deformable bodies where the motion is described by $\vec{x}(t)=\phi(t, \vec{X})$ with $\vec{x}(0)=\vec{X}$. The Lagrangian velocity is given by

$$
\vec{V}(t, \vec{X})=\frac{\partial \vec{x}}{\partial t}(t, \vec{X})=\frac{\partial \phi}{\partial t}(t, \vec{X}),
$$

while the Eulerian velocity is defined by

$$
\vec{v}(t, \vec{x})=\vec{V}\left(t, \phi^{-1}(\vec{x})\right) .
$$

The material time derivative is given by $\left.\frac{\partial}{\partial t}\right|_{\vec{X}}=\left.\frac{\partial}{\partial t}\right|_{\vec{x}}+\vec{v} \frac{\partial}{\partial \vec{x}}$, so that for any function $\psi(t, \vec{x}(t))$,

$$
\begin{aligned}
\frac{d}{d t} \psi(t, \vec{x}(t)) & =\frac{\partial \psi}{\partial \vec{x}} \frac{\partial \vec{x}}{\partial t}+\frac{\partial \psi}{\partial t} \\
& =\operatorname{grad} \psi \cdot \vec{v}+\frac{\partial \psi}{\partial t}
\end{aligned}
$$

Balance laws may be formulated in either the Lagrangian or Eulerian system. The linear momentum is defined by

$$
\begin{array}{ll} 
& \int_{\Omega} \rho(t, \vec{x}) \vec{v}(t, \vec{x}) d \omega \quad \text { (Eulerian form of momentum) } \\
\text { or } \quad & \int_{\Omega_{0}} \rho_{0}(t, \vec{X}) \vec{v}(t, \phi(t, \vec{X})) d \omega_{0} \quad \text { (Lagrangian form of momentum) }
\end{array}
$$


and the balance of linear momentum (one of Euler's laws of motion) is given (in the current configuration) by

$$
\begin{aligned}
\frac{d}{d t} \int_{\Omega} \rho(t, \vec{x}) \vec{v}(t, \vec{x}) d \omega & \equiv \int_{\Omega} \rho(t, \vec{x}) \vec{v}(t, \vec{x}) d \omega \\
& =\int_{\Omega} \rho(t, \vec{x}) \vec{f}(t, \vec{x}) d \omega+\int_{\partial \Omega} \vec{t}(\partial \Omega, \vec{x}) d a,
\end{aligned}
$$

where $\vec{f}$ is the body-force density due to the applied force and $\vec{t}$ is the contact-force density (i.e., the contact stress or traction).

Cauchy's theorem yields that a stress vector $\vec{t}$ at the point $x$ on $\partial \Omega$, which depends on the (unit) normal $\hat{n}$ to the surface at $\vec{x}$, is related to the (symmetric) Cauchy or true stress tensor $\mathbf{T}(\vec{x})$ by

$$
\vec{t}(\hat{n}, \vec{x})=\mathbf{T}(\vec{x}) \hat{n} .
$$

Using this notation, we may rewrite the linear momentum as

$$
\int_{\Omega} \rho(t, \vec{x}) \vec{f}(t, \vec{x}) d \omega+\int_{\partial \Omega} \mathrm{T}(t, \vec{x}) \hat{n} d a=\int_{\Omega} \rho \vec{v} d \omega .
$$

Applying the divergence theorem to the surface integral we obtain

$$
\int_{\partial \Omega} \mathbf{T}(t, \vec{x}) \hat{n} d a=\int_{\Omega} \operatorname{div} \mathbf{T}^{T} d \omega
$$

where div means divergence with respect to the Eulerian or current coordinate system. Hence

$$
0=\int_{\Omega}\left(\rho(t, \vec{x}) \vec{f}(t, \vec{x})+\operatorname{div} \mathbf{T}^{T}-\rho \overrightarrow{\dot{v}}\right) d \omega,
$$

which leads to Cauchy's first law of motion

$$
\rho \vec{i}=\rho \vec{f}+\operatorname{div} \mathbf{T}^{T} .
$$

This is usually coupled to $\mathbf{T}^{T}=\mathbf{T}$, and the equation of continuity: $\dot{\rho}+$ $\rho \operatorname{div} \vec{v}=0$ (conservation of mass). These are called the Eulerian field equations or the Eulerian equations of motion.

To write these equations of motion in the Lagrangian or reference coordinate systems, we must make a change of variables and the configuration gradient becomes important. Recall $B=\left(A^{-1}\right)^{T}, J=\operatorname{det} A$. The resultant contact force on the boundary $\partial \Omega$ of the current configuration $\Omega$ may be rewritten as

$$
\int_{\partial \Omega} \mathbf{T} \hat{N} d a=\int_{\partial \Omega_{0}} J \mathbf{T} \vec{B} \hat{N} d a_{0}
$$


where $\hat{N}$ is the unit outward normal to the boundary $\partial \Omega_{0}$. We then define

$$
\mathbf{S}^{T}=J \mathbf{T} \vec{B}=J \mathbf{T}\left(A^{-1}\right)^{T},
$$

where $\mathbf{S}^{T}$ is the First Piola-Kirchhoff stress tensor (it is not symmetric). Then $\mathbf{S}=J B^{T} \mathbf{T}=J A^{-1} \mathbf{T}$ is the nominal (engineering) stress tensor.

Physically, the First Piola-Kirchhoff stress tensor can be used to represent the loading in the reference configuration:

$$
\begin{aligned}
d \vec{l} & =\mathbf{T} d a \\
& =\mathbf{S}^{T} d a_{0} .
\end{aligned}
$$

Using these notions the linear momentum balance equation may be rewritten in terms of integrals over $\Omega_{0}$ and $\partial \Omega_{0}$ (i.e., with respect to the reference or Lagrangian configuration)

$$
\int_{\Omega_{0}} \rho_{0}(t, \vec{X}) \vec{f}_{0}(t, \vec{X}) d \omega_{0}+\int_{\partial \Omega_{0}} \mathbf{S}^{T}(t, \vec{X}) \hat{N} d a_{0}=\int_{\Omega_{0}} \rho_{0}(t, \vec{X}) \ddot{\phi}(t, \vec{X}) d \omega_{0} .
$$

Thus we have the Lagrangian equation of motion

$$
\operatorname{Div} \mathbf{S}+\rho_{0} \overrightarrow{f_{0}}=\rho_{0} \ddot{\phi}=\rho_{0} \ddot{u}
$$

where Div means divergence with respect to the reference coordinate system. We note that $\ddot{x}=\ddot{u}$ but $x \neq u$ ! Moreover, conservation of mass implies $\operatorname{det} A=\frac{\rho_{0}}{\rho}$.

The concepts summarized above can be readily related to the usual SEF formulations in terms of principal stretches or "extension ratios." To do this, let $\vec{M}$ be a unit vector along $d \vec{X}$ and let $\vec{m}$ be a unit vector along $d \vec{x}$. Then $\vec{m}|d \vec{x}|=A \vec{M}|d \vec{X}|$ and $|d \vec{x}|^{2}=\vec{M} \cdot A^{T} A \vec{M}|d \vec{X}|^{2}$. The ratio of the deformed length to the original length of these vectors is given by

$$
\frac{|d \vec{x}|}{|d \vec{X}|}=|A \vec{M}|=\left\{\vec{M} \cdot A^{T} A \vec{M}\right\}^{(1 / 2)} \equiv \lambda(\vec{M}),
$$

and is called the stretch in the direction $\vec{M}$ at $\vec{X}$. In the literature the $\lambda$ 's are often, in a misnomer, referred to as extension ratios. However, $\lambda(\vec{M})-1$ are the true extension ratios involving the ratio of the extension $|d \vec{x}|-|d \vec{X}|$ to the original length $|d \vec{X}|$. If $\vec{M}$ is a principal direction for $A^{T} A$, then $\lambda^{2}(\vec{M})$ is an eigenvalue for the symmetric matrix $A^{T} A$ and $\lambda(\vec{M})$ is called a principal stretch.

For a body undergoing homogeneous pure strain in the principal axis system we have that the principal stretches are eigenvalues of $A$ and (no summation)

$$
\begin{aligned}
x_{i} & =\lambda_{i} X_{i} \\
\lambda_{i} & =\frac{\partial x_{i}}{\partial X_{i}}=\frac{\partial u_{i}}{\partial X_{i}}+1 .
\end{aligned}
$$




\section{$3 \quad$ Examples}

In this section we present examples to illustrate use of the methodologies presented in Section 2 to formulate models of simple extension and generalized simple shear. In each case we will begin by formulating the model for a neo-Hookean material (SEF $U=C\left(I_{1}-3\right)$ ). In the simple extension example we will extend this to describe more general materials.

\subsection{Simple Extension}

We consider a rod under uniform extension with lateral contraction where we may begin with a choice of the SEF or with Rivlin's finite strain formulation, and use this along with the above deformation theory to derive dynamic models. We use a simple example to illustrate this: an isotropic, isochoric rubber-like rod, with a tip mass, with a finite applied stress in the direction of the principal axis $x_{1}=x$, as depicted in Figure 3. (Here, following standard convention, we use lower case letters to denote the Lagrangian coordinates.)

For incompressible neo-Hookean materials (SEF $U=\mu\left(I_{1}-3\right)$ ) the Cauchy (true) stress tensor is given by

$$
\mathbf{T}=\left[\begin{array}{ccc}
\mu \lambda_{1}^{2}-p & & 0 \\
0 & \mu \lambda_{2}^{2}-p & 0 \\
0 & 0 & \mu \lambda_{3}^{2}-p
\end{array}\right]
$$

where $p$ is an arbitrary hydrostatic stress (necessary due to the incompressibility assumption). The configuration gradient $A$ is given by

$$
A=\left[\begin{array}{ccc}
\lambda_{1} & & 0 \\
0 & \lambda_{2} & 0 \\
0 & 0 & \lambda_{3}
\end{array}\right]
$$

and thus the engineering stress tensor

$$
\mathbf{S}=\left[\begin{array}{ccc}
\mu \lambda_{1}-\frac{p}{\lambda_{1}} & & 0 \\
0 & \mu \lambda_{2}-\frac{p}{\lambda_{2}} & 0 \\
0 & 0 & \mu \lambda_{3}-\frac{p}{\lambda_{3}}
\end{array}\right]
$$

It is useful to note that, for any homogeneous pure strain deformation, the relationship between the principal stretches and the normal components of finite strain is given by

$$
\lambda_{i}^{2}=1+2 \tilde{e}_{x_{i} x_{i}}=\left(1+\frac{\partial u_{i}}{\partial x_{i}}\right)^{2} .
$$




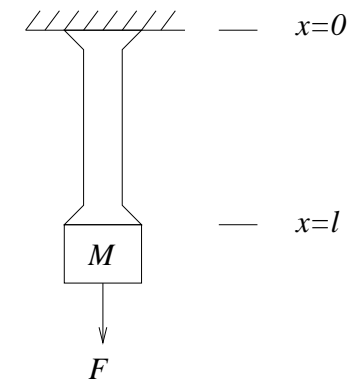

Figure 3: Rod with tip mass under tension

Thus the incompressibility condition $J=1$ reduces to $\lambda_{1}^{-1 / 2}=\lambda_{2}=\lambda_{3}$ or

$$
\left(1+\frac{\partial u_{1}}{\partial x_{1}}\right)^{-\frac{1}{2}}=\left(1+\frac{\partial u_{2}}{\partial x_{2}}\right)=\left(1+\frac{\partial u_{3}}{\partial x_{3}}\right) .
$$

In order to solve the full three dimensional problem, one would solve the three partial differential equations (with four unknowns $u_{1}, u_{2}, u_{3}$, and $p$ ) which arise from substituting $\mathbf{S}$ into the Lagrangian equation of motion (3) subject to the constraint (4).

An easier approach is to reduce the problem to a one dimensional motion by considering the deformation along the axis of the rod (which is also one of the principal axes for the deformation). Once the motion $u=u_{1}\left(t, x_{1}\right)$ along the axis of the rod has been calculated, the other motions $u_{2}$ and $u_{3}$ can be calculated using (4). Along the $x_{1}$ axis there is no deformation in the other directions in the one-dimensional formulation (i.e., $u_{2}(t)=0$, $\left.u_{3}(t)=0\right)$ and this yields the same equation as arising from setting $\mathbf{T}_{22}=$ $\mathbf{T}_{33}=0$ (which implies that $p=\frac{\mu}{\lambda_{1}}$ ). Thus the the stress tensors $\mathbf{T}, \mathbf{S}$ of (1), (2) reduce to one nontrivial component

$$
T=\mathbf{T}_{11}=\frac{E}{3}\left(\lambda_{1}^{2}-\frac{1}{\lambda_{1}}\right),
$$

and

$$
S=\mathbf{S}_{11}=\frac{T}{\lambda_{1}}=\frac{E}{3}\left(\lambda_{1}-\frac{1}{\lambda_{1}^{2}}\right) .
$$

Here $E=3 \mu$ is a generalized modulus of elasticity and we note these formulations are restricted to $\lambda_{1}>0$.

This can be used in the Lagrange formulation for longitudinal vibrations of a rubber rod with a tip mass to obtain

$$
\begin{gathered}
\rho A_{c} \frac{\partial^{2} u}{\partial t^{2}}+\gamma \frac{\partial u}{\partial t}-\frac{\partial S_{R}}{\partial x}=0 \quad 0<x<l \\
M \frac{\partial^{2} u}{\partial t^{2}}(t, l)=-\left.S_{R}\right|_{x=l}+F(t)+M g
\end{gathered}
$$


where the mass density $\rho=\rho_{o}$ since the body is isochoric, $F(t)$ is the applied external force, $A_{c}$ is the cross sectional area, $M$ is the tip mass, $g$ is the gravitational constant, $\gamma$ is the air damping coefficient, and $S_{R}$, the internal (engineering) stress resultant, is given by

$$
\begin{aligned}
S_{R} & =\frac{A_{c} E}{3}\left(\lambda_{1}-\frac{1}{\lambda_{1}^{2}}\right)+C_{D} A_{c} \frac{\partial \lambda_{1}}{\partial t} \\
& =\frac{A_{c} E}{3} \tilde{g}\left(\frac{\partial u}{\partial x}\right)+A_{c} C_{D} \frac{\partial^{2} u}{\partial t \partial x}
\end{aligned}
$$

with $\tilde{g}(\xi)=1+\xi-(1+\xi)^{-2}$. Here we have included a Kelvin-Voigt damping term $\left(C_{D}\right.$ is the Kelvin-Voigt damping coefficient) in the stress resultant $S_{R}$ as a first approximation to internal damping. This leads to the nonlinear partial differential equation IBVP

$$
\begin{aligned}
& \rho A_{c} \frac{\partial^{2} u}{\partial t^{2}}+\gamma \frac{\partial u}{\partial t}-\frac{\partial}{\partial x}\left(\frac{E A_{c}}{3} \tilde{g}\left(\frac{\partial u}{\partial x}\right)+A_{c} C_{D} \frac{\partial^{2} u}{\partial t \partial x}\right)=0, \\
& 0<x<l \\
& M \frac{\partial^{2} u}{\partial t^{2}}(t, l)=-\left.\left(\frac{A_{c} E}{3} \tilde{g}\left(\frac{\partial u}{\partial x}\right)+C_{D} A_{c} \frac{\partial^{2} u}{\partial t \partial x}\right)\right|_{x=l}+F(t)+M g \\
& u(t, 0)=0, \quad u(0, x)=\Delta(x), \quad \dot{u}(0, x)=0
\end{aligned}
$$

for dynamic longitudinal displacements of a neo-Hookean material rod in extension. In the case of small displacements, this does reduce to the usual longitudinal deformation equation for Hookean materials, which can be confirmed by considering the series expansion $\tilde{g}(\xi)=3 \xi-3 \xi^{2}+4 \xi^{3}-\ldots$ This model can also be used with a more general $\tilde{g}$ to encompass other constitutive laws (which may arise from a different SEF or from estimations made using experimental results). Identification results using experimental data with more general nonlinear constitutive laws can be found for static problems in [2] and for dynamic problems in [3]. An abstract well-posedness theoretical framework that includes systems such as (6) as well as the simple shear example of the next section is presented in [1].

\subsection{Generalized Simple Shear}

We consider a body in generalized simple shear as depicted in Figure 4. True simple shear (which is characterized by a constant angle $\gamma$ ) is rarely achieved in laboratory situations, since for most bodies the angle $\gamma$ depends on $y$. A generalization of simple shear for a neo-Hookean material provides an enlightening example when represented in terms of our continuum model. As in the simple extension example above, we use $x, y$, and $z$ for the Lagrangian coordinates. 


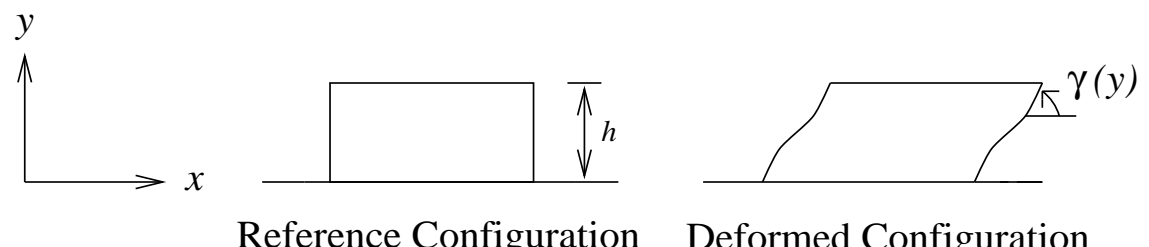

Figure 4: Simple Shear Configuration

For an incompressible, isotropic neo-Hookean material undergoing simple shear one finds [11] the Cauchy stress tensor

$$
\mathbf{T}=\left[\begin{array}{ccc}
\mu \gamma^{2}+\mu-p & \mu \gamma & 0 \\
\mu \gamma & \mu-p & 0 \\
0 & 0 & \mu-p
\end{array}\right],
$$

where $\mu$ is a material-dependent constant, $p$ is an arbitrary hydrostatic stress (as in simple extension, $p$ is necessary due to assumed incompressibility). Recall that, in general, the deformation $u(\vec{x})=\phi(\vec{X})-\vec{X}$, and (with Lagrangian coordinates $x, y, z$ )

$$
\begin{aligned}
A & =\left(\frac{\partial \phi}{\partial \vec{x}}\right) \\
& =\left(\frac{\partial u}{\partial \vec{x}}\right)+I \\
& =\left[\begin{array}{ccc}
1+\frac{\partial u}{\partial x} & \frac{\partial u}{\partial y} & \frac{\partial u}{\partial z} \\
\frac{\partial v}{\partial x} & 1+\frac{\partial v}{\partial y} & \frac{\partial v}{\partial z} \\
\frac{\partial u}{\partial x} & \frac{\partial w}{\partial y} & 1+\frac{\partial u}{\partial z}
\end{array}\right] .
\end{aligned}
$$

For simple shear

$$
A=\left[\begin{array}{lll}
1 & \gamma & 0 \\
0 & 1 & 0 \\
0 & 0 & 1
\end{array}\right]
$$

and hence $\gamma=\frac{\partial u}{\partial y}$. The engineering stress tensor is then given by

$$
\mathbf{S}=\left[\begin{array}{ccc}
\mu-p & p & 0 \\
\mu \gamma & \mu-p & 0 \\
0 & 0 & \mu-p
\end{array}\right]
$$

The Lagrangian equations of motion (3) reduce to the linear wave equation

$$
\rho \frac{\partial^{2} u}{\partial t^{2}}=\mu \frac{\partial^{2} u}{\partial y^{2}}, \quad 0<y<h
$$


with boundary conditions

$$
u(t, 0)=0, \mu \frac{\partial u}{\partial y}(t, h)=F(t)
$$

and initial conditions

$$
u(0, y)=0, \dot{u}(0, y)=0 .
$$

This interesting result (a linear wave equation for a neo-Hookean material) is consistent with the fact that the neo-Hookean SEF $\left(U=C_{1}\left(I_{1}-3\right)\right)$ is based upon a Hookean assumption in shear (see [13], [15]). The simple shear model as presented in (7) can be generalized to a nonlinear equation by including damping terms and assuming that the Cauchy stress tensor includes appropriate terms such as $\mathbf{T}_{12}=\mu \bar{g}(\gamma)$ for some nonlinear function $\bar{g}$.

For a neo-Hookean material in simple shear, the dynamics are simple linear shear waves moving up and down the thickness of the sample. Fitting neo-Hookean models to the data is equivalent to fitting Hookean models - the material will be characterized by only one parameter $\mu$ which is essentially a linear modulus of elasticity. (This will be true whether one uses a SEF approach or a dynamic approach.) If one wants to understand any of the nonlinear material properties, one should not expect to achieve this with simple shear tests using neo-Hookean material constitutive laws, or, indeed, any constitutive laws arising from a SEF of the Mooney form.

It is also interesting to note that, for simple shear, the principal stretch ratios satisfy $\lambda_{2}=\frac{1}{\lambda_{1}}$ and $\lambda_{3}=1$. Thus $I_{1}=\lambda_{1}^{2}+\lambda_{2}^{2}+\lambda_{3}^{2}=\lambda_{1}^{2}+\lambda_{1}^{-2}+1$

and $I_{2}=\lambda_{1}^{2} \lambda_{2}^{2}+\lambda_{2}^{2} \lambda_{3}^{2}+\lambda_{1}^{2} \lambda_{3}^{2}=1+\lambda_{1}^{-2}+\lambda_{1}^{2}$. Hence under simple shear the relationship $I_{1}=I_{2}$ holds. Thus simple shear is not an appropriate test for identifying coefficients for Rivlin type SEFs.

\section{Summary}

In this note we have presented one approach to the modeling of dynamic behavior of composite elastomer structures. Such structures typically involve concepts familiar to both elasticity and fluid mechanics, although elastomers do not behave like either classical elastic structures or viscous fluids. We present a methodology for model development which is based on classical large deformation formulations for deformable bodies. This methodology permits the combining of computationally tractable Lagrangian descriptions of the motion with Euler variables, such as "true stress," which arise naturally in large deformations.

To illustrate the use of this methodology we have discussed two simple but very practical examples - simple uniform extension and generalized 
simple shear. The methodology presented in these examples has been the basis of our mathematical, computational, and experimental efforts which have been reported elsewhere $[1,2,3]$.

Acknowledgments. The authors are grateful to B. Muñoz, L. Yanyo, and M. Gaitens of the Thomas Lord Research Center, Lord Corporation, and to Y. Zhang of North Carolina State University, for many helpful discussions and collaborations on the models presented here. The authors also gratefully acknowledge that this research was carried out with support in part by the U. S. Air Force Office of Scientific Research under grant AFOSR F49620-95-1-0236, and the National Science Foundation under grant NSF DMS-9508617 (with matching funds for N.J.L. from the Lord Corporation).

\section{References}

[1] H. T. Banks, D. S. Gilliam, and V. I. Shubov, Global solvability for damped abstract nonlinear hyperbolic systems, Tech. Rep. CRSCTR95-25, NCSU, 1995; Differential and Integral Equations, to appear.

[2] H. T. Banks, N. J. Lybeck, M. J. Gaitens, B. C. Muñoz, and L. C. Yanyo, Computational methods for estimation in the modeling of nonlinear elastomers, Tech. Rep. CRSC-TR95-40, NCSU, 1995; Kybernetika, to appear.

[3] - Modeling the dynamic mechanical behavior of elastomers, Tech. Rep. CRSC-TR96-26, NCSU, 1996; Rubber Chemistry and Technology, submitted.

[4] D. J. Charlton, J. Yang, and K. K. Teh, A review of methods to characterize rubber elastic behavior for use in finite element analysis, Rubber Chemistry \& Technology, 67 (1994), pp. 481-503.

[5] R. J. Crawford, Plastics and Rubbers: Engineering Design and Applications, Mechanical Engineering Publications, Ltd., London, 1985.

[6] J. D. Ferry, Viscoelastic Properties of Polymers, John Wiley \& Sons, New York, 1980.

[7] A. N. Gent, Engineering with Rubber: How to Design Rubber Components, Hanser Publishers, New York, 1992.

[8] J. E. Marsden and T. J. R. Hughes, Mathematical Foundations of Elasticity, Prentice-Hall, Englewood Cliffs, NJ, 1983.

[9] K. Nagdi, Rubber as an Engineering Material: Guideline for Users, Hanser Publishers, New York, 1993. 
[10] R. W. Ogden, Large deformation isotropic elasticity - on the correlation of theory and experiment for incompressible rubberlike solids, Proc. R. Soc. Lond. A, 326 (1972), pp. $565-584$.

[11] — Non-Linear Elastic Deformations, Ellis Horwood Limited, Chichester, 1984.

[12] R. S. Rivlin, Large elastic deformations of isotropic materials I, II, III, Phil. Trans. Roy. Soc. A, 240 (1948), pp. 459-490, 491-508, 509-525.

[13] L. R. G. Treloar, The Physics of Rubber Elasticity, Clarendon Press, Oxford, 3rd ed., 1975.

[14] K. C. Valanis and R. F. Landel, The strain-energy function of a hyperelastic material in terms of the extension ratios, Journal of Applied Physics, 38 (1967), pp. 2997-3002.

[15] I. M. Ward, Mechanical Properties of Solid Polymers, John Wiley \& Sons, New York, 2nd ed., 1983. 